\title{
Non-monotonic behaviour of the superconducting order parameter in Nb/PdNi bilayers observed through point contact spectroscopy
}

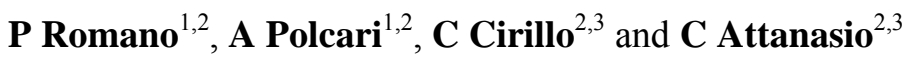 \\ ${ }^{1}$ Dipartimento di Scienze per la Biologia, la Geologia e l'Ambiente, Università del \\ Sannio, via Port'Arsa 11, 82100 Benevento, Italy. \\ ${ }^{2}$ CNR-SPIN Salerno, via Ponte Don Melillo, 84084 Fisciano (SA), Italy. \\ ${ }^{3}$ Dipartimento di Fisica "E.R. Caianiello" Università di Salerno, via Ponte Don Melillo, \\ 84084 Fisciano (SA), Italy.
}

E-mail: promano@unisannio.it

\begin{abstract}
Point contact spectroscopy measurements have been performed on $\mathrm{Nb} / \mathrm{PdNi}$ bilayers in which the thickness of the $\mathrm{Nb}$ layer, $\mathrm{d}_{\mathrm{Nb}}$, was kept constant to $40 \mathrm{~nm}$ while the thickness of PdNi, $\mathrm{d}_{\mathrm{PdNi}}$, was changed from $2 \mathrm{~nm}$ to $9 \mathrm{~nm}$. Features related to the superconducting gap induced in the ferromagnet have been observed in the $\mathrm{dV} / \mathrm{dI}$ versus $\mathrm{V}$ curves. These structures show a nonmonotonic behaviour as a function of $\mathrm{d}_{\mathrm{PdNi}}$ as a consequence of the damped oscillatory behaviour of the superconducting order parameter in the ferromagnetic layer.
\end{abstract}

PACS. 74.45.+c; 74.78.Fk; 75.70.Cn. 


\section{Introduction}

Recently great attention has been devoted to the coupling between superconductivity and ferromagnetism in Superconducting/Ferromagnetic (S/F) thin film hybrids [1,2] due to the rich physics originated from the coexistence of two competing orderings: in $\mathrm{S}$ the electrons with antiparallel spin are coupled to form Cooper pairs, while in $\mathrm{F}$ the exchange field, $\mathrm{E}_{\mathrm{ex}}$, forces the spin in a parallel configuration. For this reason a strong reduction of the order parameter in $\mathrm{S} / \mathrm{F}$ hybrids is expected, since $\mathrm{E}_{\mathrm{ex}}$ will try to align the spins in the Cooper pair, leading to a strong pair breaking effect. Indeed, the proximity effect picture at the $\mathrm{S} / \mathrm{F}$ interface is strongly modified compared to the $\mathrm{S} / \mathrm{N}$ case (here $\mathrm{N}$ stands for normal metal). It is well known that the mechanism responsible of the proximity effect phenomenon at $\mathrm{S} / \mathrm{N}$ interface is the Andreev reflection [3]. What happens is that electrons from the $\mathrm{N}$ side with energy lower than the superconducting gap, $\Delta$, cannot penetrate into the S side. However, an incoming electron can be transferred into the superconductor if a second electron is also transferred through the interface, thus forming a Cooper pair in S and creating a hole in $\mathrm{N}$ [3]. Electrons and holes will move in opposite directions, adding to the conductance of the normal electrode, but they will loose their coherence during the propagation in the metal. The Andreev pair disappears over a characteristic length $\xi_{\mathrm{N}}$, which measures how far the two electrons leaking from the superconductor will diffuse in phase. In the dirty limit $\xi_{N}=\left(\hbar D_{N} / 2 \pi E\right)^{1 / 2}$, where $D_{N}$ is the diffusion coefficient of the normal metal and $\mathrm{E}$ is the energy responsible of the de-phasing. In a normal metal $\mathrm{E}$ is the thermal energy, $\mathrm{k}_{\mathrm{B}} \mathrm{T}$, leading to $\xi_{\mathrm{N}}=\left(\hbar \mathrm{D}_{\mathrm{N}} / 2 \pi \mathrm{k}_{\mathrm{B}} \mathrm{T}\right)^{1 / 2}$. Andreev reflection thus contributes to an increasing of the conductance in the under-gap region, which will be well recognized in the $\mathrm{dI} / \mathrm{dV}$ vs $\mathrm{V}$ curves. For example, in case of resistive contact, the conductance below the gap voltage $(\Delta / \mathrm{e})$ becomes twice the normal conductance due to Andreev reflections. In the Blonder, Tinkham and Klapwijk (BTK) model [4], the conductance curves will show a characteristic bell-shaped behaviour with the gap value corresponding to the voltage where the conductance increases, at zero temperature, up to a maximum value of twice the background value. In other words, the differential resistance $\mathrm{dV} / \mathrm{dI}$ of a pure resistive contact below the gap voltage becomes half of the normal resistance. In the model, an insulating (I) barrier mimics the properties of the interface between $\mathrm{S}$ and $\mathrm{N}$, which strength can be progressively increased going from zero (pure resistive contact) up to reach a value that allows an appreciable tunneling current between $\mathrm{S}$ and $\mathrm{N}$. As the barrier strength is increased the conductance will indeed show a double-peak structure at $\pm \Delta / \mathrm{e}$, as in tunnel junctions, or double-dips in the resistance. In the case of an S/F boundary the situation is complicated due to energy splitting of the spin-up and 
spin-down sub-bands in the ferromagnet, which is responsible of a strong reduction of the reflections, since not all the majority spin electrons at the S/F boundary will be able to find a matching electron with opposite minority spin. For this reason the Andreev reflections are totally suppressed in fully spin polarized metal [5]. In addition, in S/F systems the coherence length, $\xi_{\mathrm{F}}$, is strongly reduced. In this case, in fact, the incoming electron and the Andreev reflected hole occupy opposite spin bands. Consequently the induced superconducting order parameter disappears in F over a much shorter distance, this time controlled by the strength of the ferromagnet, namely, in the dirty limit, $\xi_{\mathrm{F}}=\left(\hbar \mathrm{D}_{\mathrm{F}} / \mathrm{E}_{\mathrm{ex}}\right)^{1 / 2}$, where $\mathrm{D}_{\mathrm{F}}$ is the diffusion coefficient of the ferromagnet. Moreover, due to the presence of the exchange field a spatial oscillation of the order parameter is superimposed on its decay in F [6].

The aim of this work is to probe this inhomogeneous character of the superconducting order parameter in S/F hybrids through Point Contact Spectroscopy (PCS). This technique has been widely used in the past to study the $\mathrm{S}$ electronic properties: mechanically pushing a tip, generally made of normal metal N, on the top of a superconductor, S-N contacts can be realized [7]. It is important to remind that the contact between tip and sample can also generate S-I-N and S-I-S contacts, when a suitable barrier, either natural or artificial, is present between the two electrodes [8-11]. From the I-V behaviour, and more specifically from the $\mathrm{dI} / \mathrm{dV}$ curves, it is possible to estimate the basic properties of a superconductor, as the energy gap value and the density of states, as well as the symmetry of the order parameter in the case of unconventional superconductors, being the experiment sensitive to both the magnitude and the phase of the order parameter [7]. While for the high- $\mathrm{T}_{\mathrm{c}}$ cuprates, PCS provided the earliest measurements of the superconducting gap spectra [8], more recently it also appeared to be a powerful tool in investigating the superconducting order parameter even in the case of multiple gaps [12]. Moreover, using a superconducting tip, S-F contacts have been successfully employed to determine the spin polarization of several ferromagnets [13]. PCS technique has also be extended to study proximized structures, like S/F bilayers, obtaining S/F-N contacts between the bilayer and the normal metal tip [14]. Since in this case two different interfaces are involved, namely between $\mathrm{S}$ and $\mathrm{F}$, and between $\mathrm{F}$ and $\mathrm{N}$, more complex conductance curves may be obtained. The BTK model should then include these processes, as well as the presence of polarized electrons due to F. For all these reasons, while PCS can be simple in ideal situation, in real experimental conditions its application, as well as the data interpretation, can be quite complicated.

In this paper we study the simplest $\mathrm{S} / \mathrm{F}$ hybrids, namely $\mathrm{S} / \mathrm{F}$ bilayers. For these structures a nonmonotonic behaviour of the critical temperature, $\mathrm{T}_{\mathrm{c}}$, over $\xi_{\mathrm{F}}$ as a function of the thickness of the 
ferromagnetic layer, $d_{F}$, has been found theoretically as well as experimentally $[1,15-20]$. The superconducting transition temperature is the simplest parameter which reveals intriguing behaviour typical of $\mathrm{S} / \mathrm{F}$ structures, but the inhomogeneous character of the order parameter in the ferromagnetic layer also affects the characteristics of S/F/S Josephson junctions [21], as well as the density of states (DOS) in S/F based tunnel junctions [22,23]. Following this approach, we present PCS measurements realized between a normal metal Au tip on $\mathrm{Nb} / \mathrm{PdNi}$ bilayers with different PdNi thickness, PdNi being a weakly ferromagnetic alloy. We observe that some features are present in the $\mathrm{dV} / \mathrm{dI}$ versus $\mathrm{V}$ curves which are related to the superconducting gap induced in the ferromagnet as well as to the $\mathrm{Nb}$ order parameter. Both these structures show a non-monotonic behaviour as a function of $\mathrm{d}_{\mathrm{PdNi}}$.

\section{Fabrication}

The S/F bilayers consist of a 40-nm thick $\mathrm{Nb}$ layer, and of a weakly ferromagnetic alloy, $\mathrm{Pd}_{0.84} \mathrm{Ni}_{0.16}(=\mathrm{PdNi})$ layer, with variable thickness, $\mathrm{d}_{\mathrm{PdNi}}=2-4-5-9 \mathrm{~nm}$. This system was chosen since the existence of an inhomogeneous superconducting order parameter in $F$, peculiar characteristic of $\mathrm{S} / \mathrm{F}$ hybrids $[1,2,6,15,16]$, has been already demonstrated for $\mathrm{Nb} / \mathrm{Pd}_{1-\mathrm{x}} \mathrm{Ni}_{\mathrm{x}}$ systems [19-22,24-26]. Moreover, PdNi is characterized by longer spin-flip scattering length compared to the widely used CuNi alloy [27]. The bilayers have been deposited by a threetarget UHV dc magnetron sputtering, equipped with a load-lock chamber. The base pressure in the main chamber was in the low $10^{-8}$ mbar range. The $\mathrm{Nb}$ and PdNi layers have been grown on $\mathrm{Al}_{2} \mathrm{O}_{3}$ substrates at typical power of $\mathrm{W}_{\mathrm{Nb}}=390$ Watt and $\mathrm{W}_{\mathrm{PdNi}}=90$ Watt, and Argon pressure of 3 $\mu$ bar and $8 \mu$ bar, for $\mathrm{Nb}$ and PdNi respectively. During the deposition the substrate holder was kept at $\mathrm{T}=100{ }^{\circ} \mathrm{C}$. These fabrication conditions determine the deposition rates $\mathrm{r}_{\mathrm{Nb}}=2.7 \mathrm{~nm} / \mathrm{s}$ and $\mathrm{r}_{\mathrm{PdNi}}=2.2 \mathrm{~nm} / \mathrm{s}$, respectively, which were controlled with a thickness monitor calibrated by low angle X-Ray reflectivity measurements. The Ni content of the ferromagnetic alloy, $\mathrm{x}=0.16$, has been determined by Energy Dispersion Spectroscopy measurements. As reported in detail in Ref. [25] for this composition, the ordering temperature and the exchange energy of the alloy are $\mathrm{T}_{\text {Curie }}=190 \mathrm{~K}$ and $\mathrm{E}_{\mathrm{ex}} \approx 14 \mathrm{meV}$, respectively. Using for the diffusion coefficient in the ferromagnet $D_{F}=2.3 \times 10^{-4} \mathrm{~m}^{2} / \mathrm{s}$ [25] we have a penetration of the Cooper pairs inside the $\mathrm{F}$ layer $\xi_{\mathrm{F}}$ of the order of 3-4 $\mathrm{nm}$. 


\section{Preliminary characterization}

The bilayers have been preliminary characterized by transport measurements. In particular the superconducting transition temperature was resistively measured using a standard dc four-probe technique. $\mathrm{T}_{\mathrm{c}}$ was defined at the midpoint of the $\mathrm{R}(\mathrm{T})$ transition. The transitions width, defined as $\Delta \mathrm{T}_{\mathrm{c}}=\mathrm{T}_{\mathrm{c}}\left(0.9 \mathrm{R}_{\mathrm{N}}\right)-\mathrm{T}_{\mathrm{c}}\left(0.1 \mathrm{R}_{\mathrm{N}}\right)$, where $\mathrm{R}_{\mathrm{N}}$ is the value of the resistance in the normal state, never exceeded $50 \mathrm{mK}$. The single $\mathrm{Nb}$ film, $40 \mathrm{~nm}$ thick, has a superconducting temperature $\mathrm{T}_{\mathrm{cS}}=8.2$ $\mathrm{K}$, a low temperature resistivity $\rho_{\mathrm{Nb}}=12 \mu \Omega \mathrm{cm}$, and a superconducting coherence length, estimated from perpendicular upper magnetic field, $\xi_{\mathrm{S}}=6 \mathrm{~nm}$. The dependence of $T_{c}$ as a function of the PdNi layer thickness in $\mathrm{Nb} / \mathrm{PdNi}$ bilayers is reported in figure 1. The critical temperature rapidly decreases with increasing the thickness of the ferromagnetic layer, showing a minimum around $\mathrm{d}_{\mathrm{PdNi}} \approx 5 \mathrm{~nm}$. The value of the dip position scales reasonably well with the ones obtained for $\mathrm{Nb} / \mathrm{PdNi}$ systems for different $\mathrm{Ni}$ concentrations of the alloy $[19,20,24]$. Despite the small number of samples, this result confirms the non-monotonic behaviour of $\mathrm{T}_{\mathrm{c}}\left(\mathrm{d}_{\mathrm{PdNi}}\right)$, the difference in the critical temperature between the sample with $\mathrm{d}_{\mathrm{PdNi}}=5 \mathrm{~nm}$ and the saturation value being in fact $\Delta \mathrm{T} \approx 0.14 \mathrm{~K} \gg \Delta \mathrm{T}_{\mathrm{c}}$. As discussed in the following section, the signatures of the non-homogeneous superconducting order parameter were further investigated below $\mathrm{T}_{\mathrm{c}}$ by PCS technique.

\section{Point contact measurements}

In order to infer the electronic properties in our samples, we have performed point contact measurements making possible the process of Andreev reflections between a normal tip and the bilayer. A tip made of normal metal $(\mathrm{Au})$ has been pushed on the ferromagnetic side of a S/F bilayer. Since $\mathrm{Au}$ is a soft material, a direct contact with $\mathrm{Nb}$ can be excluded, the tip flattening rather than damaging the PdNi layer. To shed light on the effects of the tip-sample interaction Scanning Electron Microscopy (SEM) analyses have been performed. The results (not reported here) reveal in fact no sign of degradation and/or presence of holes in the PdNi surface. The I-V curves of the contact were recorded while the junction was current biased and the gap value was obtained from the numerically calculated resistance $\mathrm{dV} / \mathrm{dI}$ for each sample. A sketch of the spatial dependence of the order parameter, $\Psi(x)$, in S/F bilayers for two different values of $d_{F}$ is reported in figure 2, where also the point contact geometry is illustrated. The Andreev spectroscopy measurements should be performed in the ballistic limit, namely for values of the 
point contacts size much smaller than the carrier mean free path. The point contact diameter, $a$, can be evaluated by means of the Sharvin resistance in the ballistic regime as [28]

$$
R=4 \rho l / 3 \pi a^{2}
$$

where $\rho$ and $l$ are the low temperature resistivity and mean free path of the sample. In our case the measured value of $\rho$ was equal to $13 \mu \Omega \mathrm{cm}$ for all the investigated bilayers. Since this value is very close to the measured low temperature resistivity for a single $\mathrm{Nb}$ film $\left(\rho_{\mathrm{Nb}}=12 \mu \Omega \mathrm{cm}\right)$ we assume that $\rho_{\mathrm{Nb} / P d N i} l_{N b / P d N i}=3.72 \times 10^{-6} \mu \Omega \mathrm{cm}^{2}$, value which is valid for single $\mathrm{Nb}$ [29]. Within this limit we obtain $l=3 \mathrm{~nm}$. The resistance of the contacts in our samples, of the order of few Ohms, gives from Eq. (1) a diameter ranging from 10 to about $100 \mathrm{~nm}$, larger than the mean free path of carriers in the bilayer. Therefore, this large contact area can cause many other effects as diffusive transport, multiple contacts and proximity effects [30], which are not intrinsic features of the samples and can affect the spectra. On the other hand, the Sharvin formalism rigorously applies for clean systems [31], while in dirty superconductors with extremely low $l$ values the condition $a<l$ is hardly achieved. When the contact resistance is measured, this could just reflect in a higher effective barrier strength but still in the framework of a ballistic transport [31].

In figure 3 the differential resistances numerically calculated from the $\mathrm{I}-\mathrm{V}$ curves of three samples with different ferromagnetic thickness, $d_{F}$, are shown below $T_{c}$. The corresponding reduced temperature, $t=T / T_{c}$, is indicated for each curve. The general behaviour is characterized by two components at low bias: a sharp single dip at zero and double symmetric dips indicated in figure by the red arrows. The zero-bias dip, known as zero-bias anomaly (ZBA), has also been observed in different superconducting systems such as $\mathrm{Nb}-\mathrm{Ag}$ (or $\mathrm{Al}$ ) microjunctions [32] and microshort $\mathrm{Au}-\mathrm{YBa}_{2} \mathrm{Cu}_{3} \mathrm{O}_{7-\delta}$ junctions [33]. Most notably, ZBA seems to appear more systematically in the Andreev spectra with small contact resistances and thus with large contact sizes, indicating that it can be related to the contact geometry and not an intrinsic feature of the sample in question. Various theoretical models have been proposed, like ordinary proximity effects [33] or multiple Andreev reflections [34], both predicting easy suppression of the effect by a small applied magnetic field. In our samples, the ZBA decreases with a magnetic field applied perpendicularly to the sample, as shown for example in figure $4 \mathrm{a}$ for a contact realized on the sample with $d_{F}=9 \mathrm{~nm}$ at $\mathrm{T}=2.5 \mathrm{~K}$. The zero-bias conductance normalized to the background conductance, $\mathrm{G}(0)_{\text {norm, }}$, versus the applied magnetic field is shown in figure $4 \mathrm{~b}$ : 
$\mathrm{G}(0)_{\text {norm }}$ rapidly decreases down to $0.04 \mathrm{~T}$, a value comparable with the coercive field of PdNi [25], and totally disappears already at $0.1 \mathrm{~T}$. The rapid reduction at low field indicates that $\mathrm{Nb}$ is not responsible for this behaviour, being the typical upper critical magnetic field values at this temperature for our samples of the order of $1 \mathrm{~T}$ [24]. On the contrary, it seems reasonable to ascribe this dependence to the occurrence of crossed Andreev reflections from multiple $\mathrm{F}$ domains close to the $\mathrm{S} / \mathrm{F}$ interface [35]. On the other hand, as shown in figure 5 for two different contacts realized on the same sample, the ZBA disappears in zero-field when the temperature is increased up to $4.2 \mathrm{~K}$, value which is smaller than the critical temperature of the sample. This behaviour is probably due to the local nature of the PCS technique, which is only able to probe a surface portion of the sample. It is worth noting that, regardless its origin, in some cases the ZBA can overwhelm the entire conductance spectrum and render the gap measurements unfeasible [36]. In our case, the ZBA might be related to a change of phase of the order parameter in F. When, in fact, the order parameter changes sign along the electron's trajectory, a zero-bias conductance peak can appear due to Andreev surface bound states (ABS). This feature, absent in BTK's calculation, is for instance expected for the case of a gap having a dwave symmetry, as Kashikawa et al. have demonstrated for YBCO [37]. Even more relevant for our work, the presence of ABS is expected also for S/F systems [38].

Turning our attention to the $\mathrm{dV} / \mathrm{dI}$ data, the dips just above the ZBA have been related to the superconducting gap voltage in S-N microjunctions [32], as expected in the BTK model. In our case, the double dip structure could be the signature of the superconducting gap induced in the $\mathrm{F}$ layer, for this reason much lower than the $\mathrm{Nb}$ gap value. In figure 6 the double voltage dip position $V_{\text {dip }}$ evaluated from figure 3 is shown as a function of $d_{F}$. The value for $d_{F}=0$ has been calculated from the $T_{c}$ of pure $\mathrm{Nb}$ through the relation $2 \Delta / \mathrm{k}_{\mathrm{B}} \mathrm{T}_{\mathrm{c}}=3.52$. Although for the sample with $d_{F}=2 \mathrm{~nm}$ the value of reduced temperature is different $(t=0.56)$, the dip position can still be reasonably related to the zero-temperature gap voltage, considering the temperature dependence of the superconducting gap in conventional materials [39]. A non-monotonic behaviour of $2 V_{\text {dip }}\left(d_{F}\right)$ is observed, very close reminding the $T_{c}\left(d_{F}\right)$ dependence of figure 1 (reported again in figure 6 for sake of clarity), with a clear minimum at $d_{F}=5 \mathrm{~nm}$ which represents the experimental confirmation of the change of sign of the superconducting order parameter at the $\mathrm{F} / \mathrm{I}$ interface ( $\mathrm{I}$ is the vacuum) $[15,38]$. The additional resistance dips sometimes observed in the low bias region at voltages $\mathrm{V}_{\mathrm{n}}$, probably arise from $\mathrm{n}$-fold Andreev reflection process [39], or from multiple scattering and impurities [40].

Finally, in the $\mathrm{dV} / \mathrm{dI}$ data presented in figure 3 one more feature can be observed, namely a resistance double peak in the high bias region which appears stable and symmetric around zero 
(black arrows in the figure) in all the samples. Similar features have been observed on S-N microcontacts [32] as well as in heterojunctions involving contacts between a superconductor and a semiconductor [41]. Although their origin is not completely clear, these structures, appearing like minima in the conductance of Andreev contacts, have been explained in terms of proximity-induced superconductivity [42]. In figure 7 we report the double voltage peaks position, $\mathrm{V}_{\text {peak }}$, as a function of $\mathrm{d}_{\mathrm{F}}$, which shows, once again, a non-monotonic behaviour, even though in this case, probably due to the few experimental points, the minimum of the curve appears at $\mathrm{d}_{\mathrm{F}}=2 \mathrm{~nm}$. We can also estimate the critical temperature dependence, $\mathrm{T}_{\mathrm{c}}{ }^{2 \mathrm{Vpeak}}\left(\mathrm{d}_{\mathrm{F}}\right)$, from the position of the dip through the relation $2 \mathrm{~V}_{\text {peak }} / \mathrm{k}_{\mathrm{B}} \mathrm{T}_{\mathrm{c}}{ }^{2 \mathrm{~V} \text { peak }}=3.52$. As it is shown in the inset of figure 7 the values for $\mathrm{T}_{\mathrm{c}}{ }^{2 \mathrm{~V} \text { peak }}$ are slightly different from the values of the critical temperature measured from transport measurements. We believe that this discrepancy is again due to the fact that PCS is a local measurement while resistive transitions probe the average superconducting order parameter over the whole sample.

\section{Discussion and Conclusions}

$\mathrm{Nb} / \mathrm{PdNi}$ bilayers for different values of PdNi thickness have been analyzed. The $\mathrm{T}_{\mathrm{c}}$ of the bilayers rapidly decreases with increasing the PdNi thickness, showing a minimum for $\mathrm{d}_{\mathrm{PdNi}}$ of about $5 \mathrm{~nm}$. The non-monotonicity exhibits itself also in PCS measurements. In fact, a nonmonotonic behaviour of the gap induced in the ferromagnet, connected with a resistance double dip structure, is observed. A similar result is found for the proximized $\mathrm{Nb}$ order parameter, connected with a resistance double peak structure, as a function of $\mathrm{d}_{\mathrm{PdNi}}$. The conventional BTK theory [4] cannot help as it is to model the data, since it analyzes the conductance versus bias voltage curves for nonmagnetic metal-superconductor contacts. In our samples, the superconductor has been substituted by a S/F bilayer, making the situation somewhat different. In this case, in fact, proximity can occur between $\mathrm{S}$ and $\mathrm{F}$. As a consequence, new features can appear in the data, which can be accounted only through a modification of the model, for instance including two gaps in the conductance expression, one for the Andreev process and one for the quasiparticle transport [42]. Moreover, the spin polarization of the ferromagnet should also be considered in the model; as a further consequence, the normalized conductance at zero bias will be lower than 2, as instead expected for pure Andreev reflections, due to an imbalance in the number of spin-up and spin- down electrons which can cause a suppression of the Andreev reflection probability. In the data, we have also observed a ZBA in the form of a zero bias dip. This feature, absent in BTK's calculations, might be explained in terms of a change of sign of the order parameter along the electron's trajectory. For high- $\mathrm{T}_{\mathrm{c}} \mathrm{YBCO}$ superconductor, 
for instance, it has been shown that a ZBA can come from an unconventional gap symmetry (dwave) [37], due to the fact that the quasiparticles may experience a sign change of the order parameter. The general consequence is the development of ABS in the quasiparticle excitation spectrum at the Fermi energy. These bound states exist only at the interface but do not have important effect on the overall measured spectrum, leading to a ZBA in certain geometries. The BTK model can thus be extended in order to describe these novel effects. In the system under study the ZBA can originate from a change of sign of the order parameter at the F/I interface [38]. Despite the small number of the analyzed samples, the experimental data presented in this work confirm the change of sign of the superconducting order parameter at the S/F interface as a function of the ferromagnetic layer thickness. More work is in progress in order to further analyze our data by means of appropriate models, taking into account the new features appearing in the resistance curves and the role of the ferromagnet, in the framework of the interactions between superconductivity and ferromagnetism.

\section{Acknowledgements}

The authors wish to thank Dr. R. Fittipaldi for performing SEM analyses. 


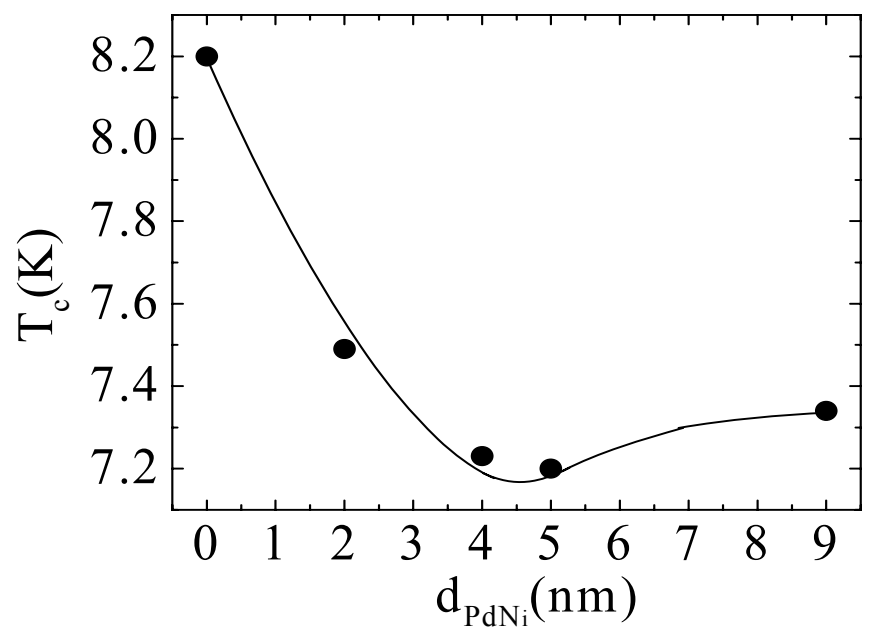

Figure 1. Superconducting critical temperature, $T_{c}$, as a function of the PdNi thickness, $d_{P d N i}$. The line is a guide to the eye. 


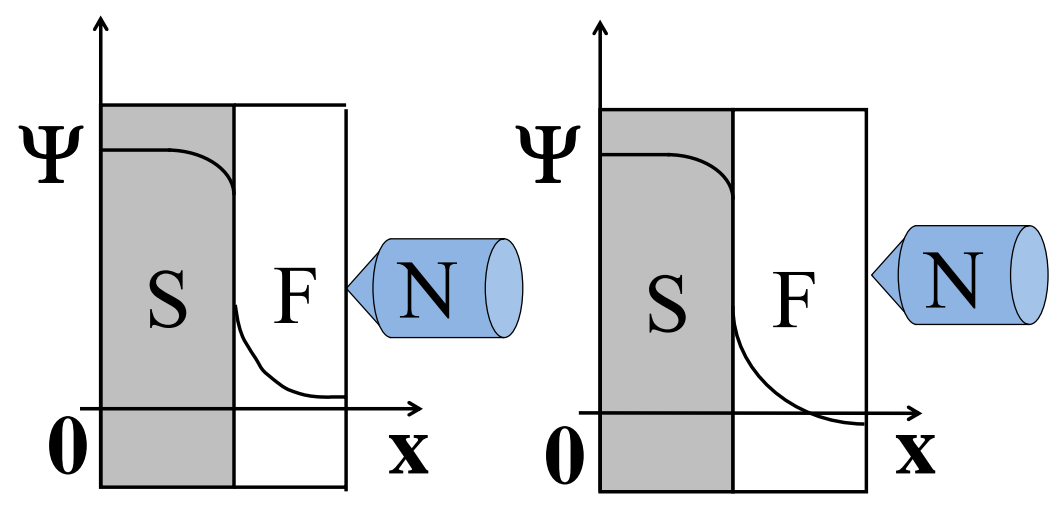

Figure 2. (Color online) Sketch of the spatial dependence of the order parameter, $\Psi(\mathrm{x})$, in S/F bilayers for two different values of $d_{F}$ (adapted from Ref. [15]). Depending on the ferromagnetic layer thickness, the sign of the order parameter at the $\mathrm{F}$ free surface may change from positive to negative. A scheme of the point contact measurement geometry is also illustrated. The whole sketch is not in scale. 


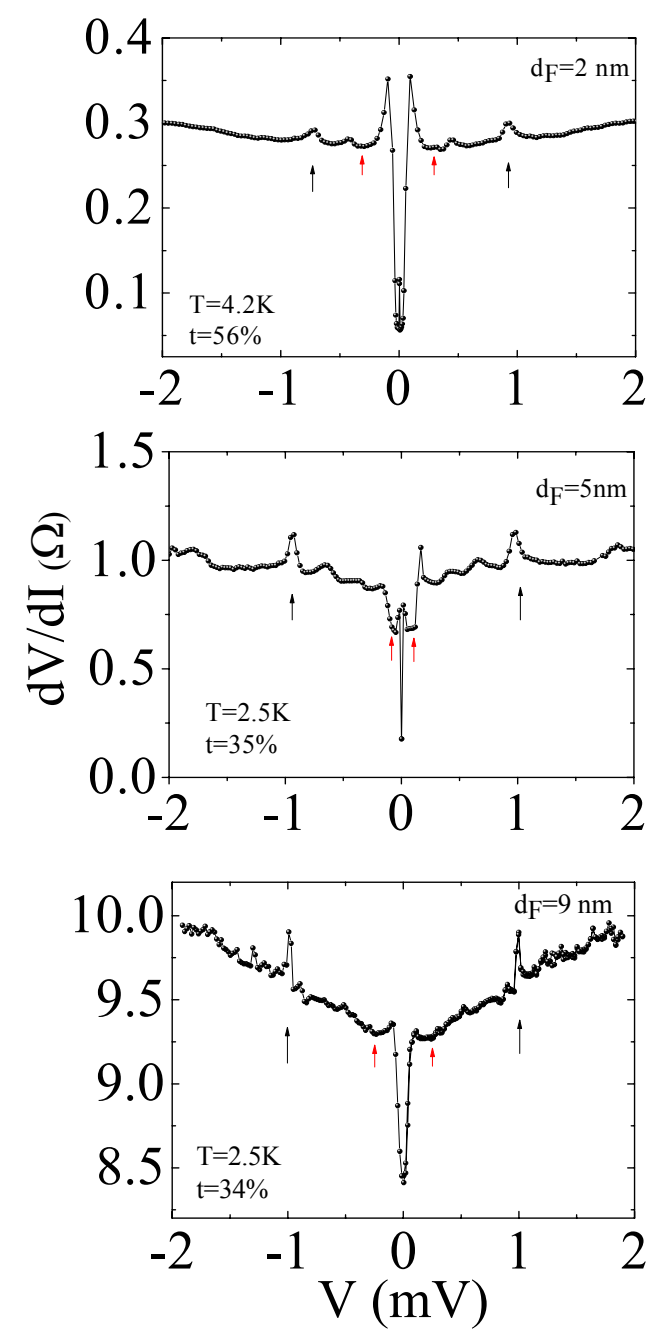

Figure 3. (Color online) Differential resistances, $d V / d I$, for samples with different thickness of the PdNi layer, $\mathrm{d}_{\mathrm{F}}$, at different reduced temperatures, $\mathrm{t}=\mathrm{T} / \mathrm{T}_{\mathrm{c}}$. The red arrows indicate a double symmetric dip just above the ZBA. The black arrows indicate a double symmetric peak at higher biases. 

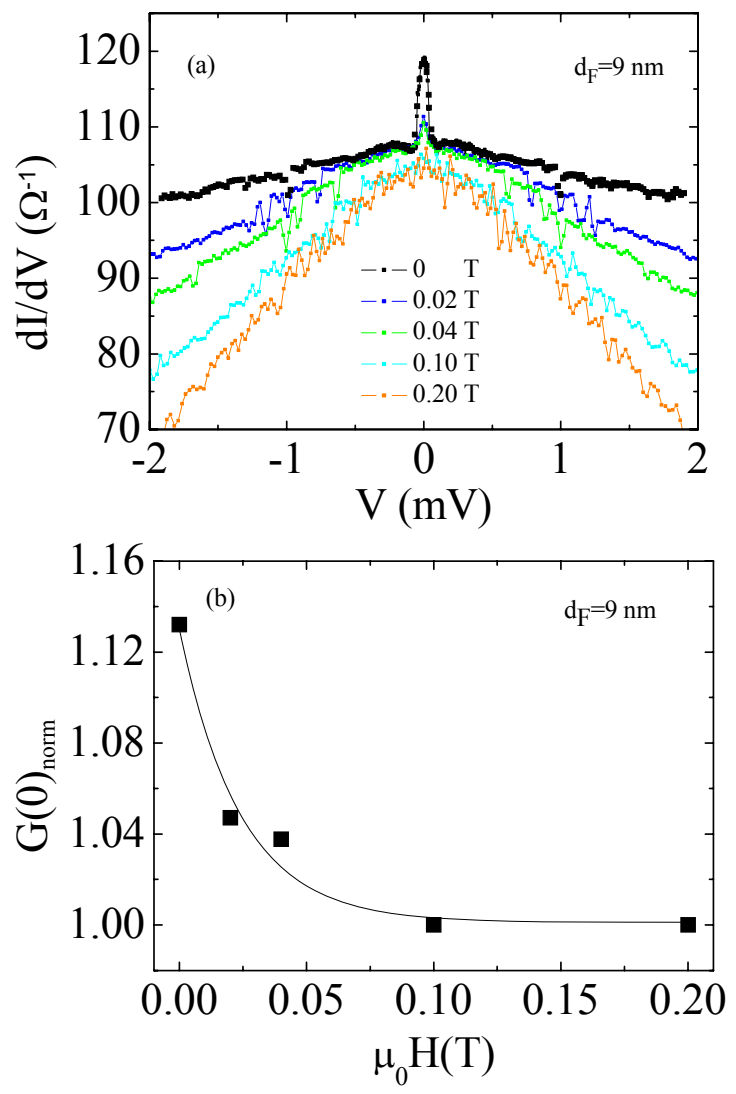

Figure 4. (Color online) The conductance of a contact realized on the sample with $d_{F}=9 \mathrm{~nm}$ at different fields. b) The normalized zero-bias conductance as a function of the applied magnetic field. 


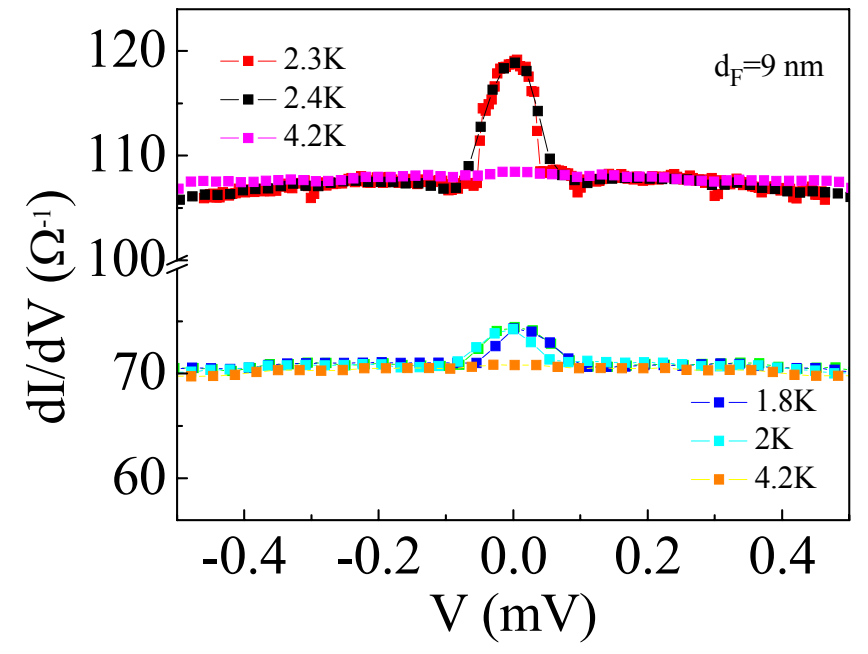

Figure 5. (Color online) The conductance of two different contacts realized on the sample with $d_{F}=$ $9 \mathrm{~nm}$ at different temperatures. 


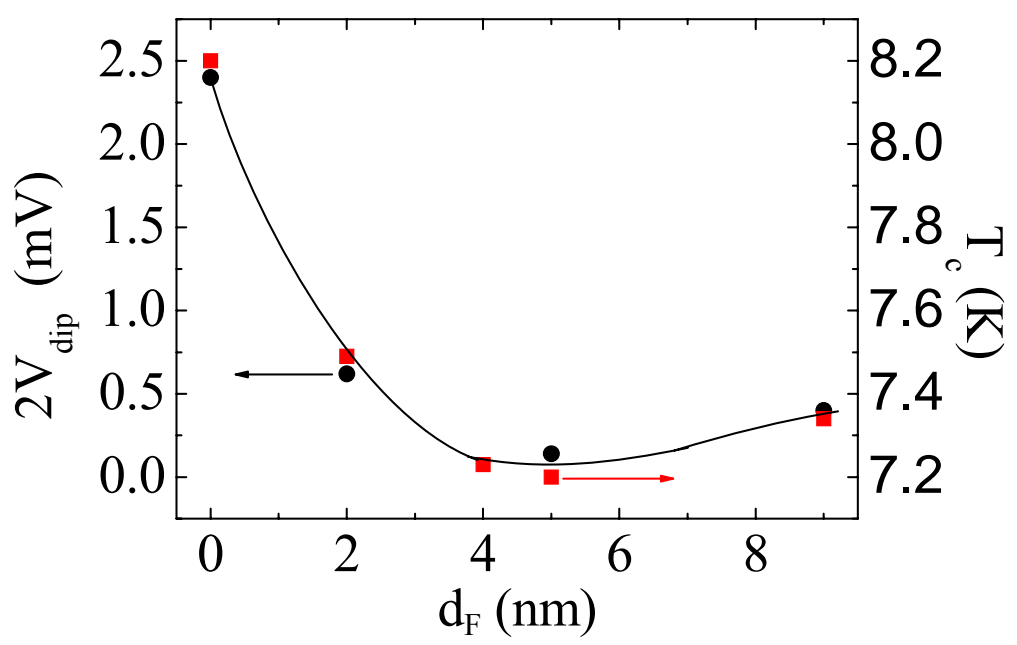

Figure 6. (Color online) Thickness dependence of the low bias resistance double dip position as indicated by the red arrows in figure 3 (left scale) and of the critical temperature values (right scale). The line is a guide to the eye. 


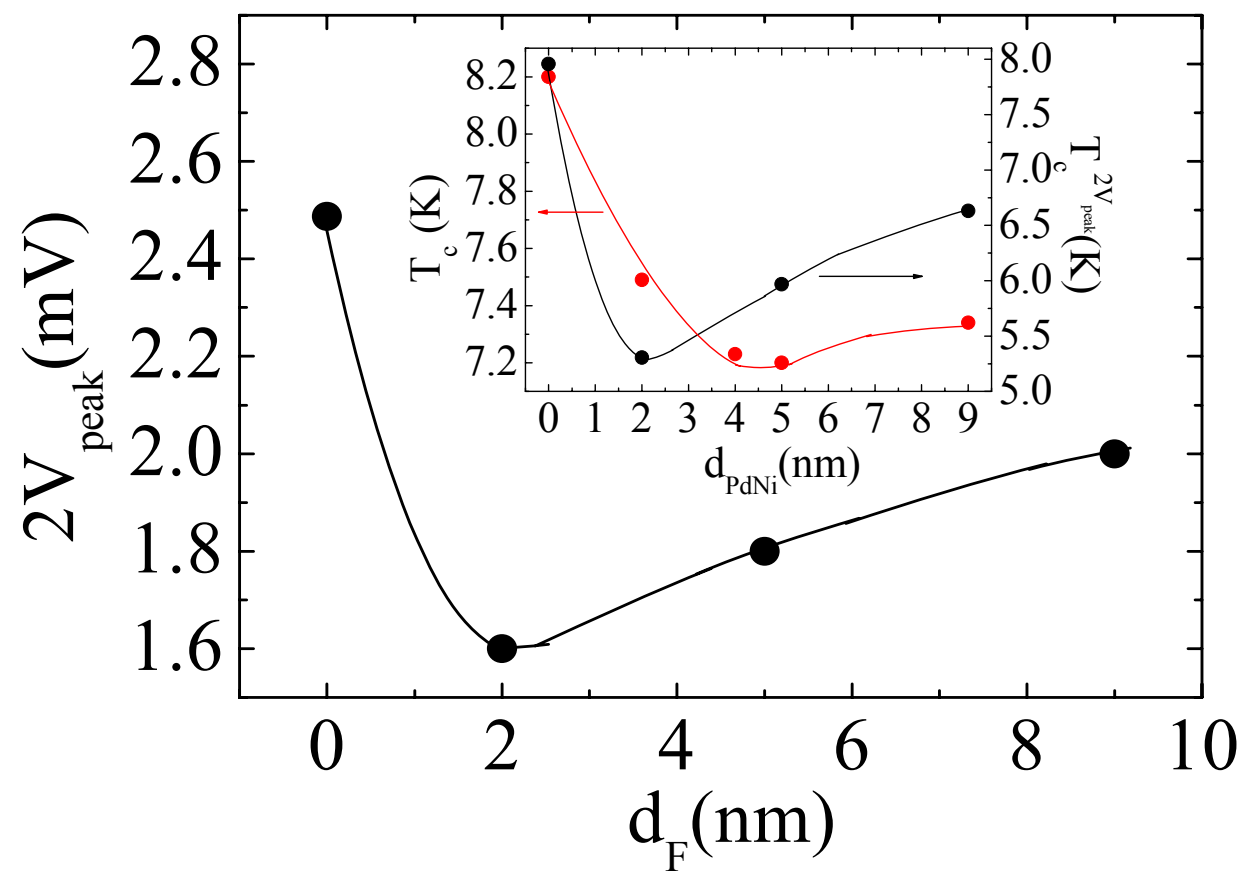

Figure 7. (Color online) High bias resistance double peak position as a function of $\mathrm{d}_{\mathrm{F}}$ (see black arrows in figure 3). Inset: comparison between the critical temperature values estimated from the high bias double peak position (right scale) and from the R(T) measurements (left scale).

The lines are guide to the eye. 


\section{References}

[1] Buzdin A I 2005 Rev. Mod. Phys. 77935

[2] Bergeret F S, Volkov A F and Efetov K B 2005 Rev. Mod. Phys. 771321

[3] Andreev A F 1964 Zh. Eksp. Teor. Fiz. 461823 [1964 Sov. Phys. JETP 19 1228]

[4] Blonder G E, Tinkham M and Klapwijk T M 1982 Phys. Rev. B 254515

[5] de Jong M J M and Beenakker C W J 1995 Phys. Rev. Lett. 741657

[6] Demler E A, Arnold G B and Beasley M R 1997 Phys. Rev. B 5515174

[7] Deutscher G 2005 Rev. Mod. Phys. 77109

[8] Zasadzinski J 2003 in The Physics of Superconductors, Ed. by Bennemann K H and Ketterson J B (Springer, Berlin)

[9] Huang Q, Zasadzinski J F , Gray K E, Richards D R and Hinks D G 1990 Appl. Phys. Lett. 57 2356

[10] DeWilde Y, Miyakawa N, Guptasarma P, Iavarone M, Ozyuzer L, Romano P, Hinks D G, Kendziora C, Crabtree G W and Gray K E 1998 Phys. Rev. Lett. 80153

[11] Romano P, Chen J and Zasadzinski J 1998 Physica C 29515

[12] Szabó P, Samuely P, Kacmarcík J, Klein T, Marcus J, Fruchart D, Miraglia S, Marcenat C and Jansen A G M 2001 Phys. Rev. Lett. 87 137005; Chen T Y, Tesanovic Z, Liu R H, Chen X H, and Chien C L 2008 Nature 4531224

[13] Soulen R J Jr, Byers J M, Osofsky M S, Nadgorny B, Ambrose T, Cheng S F, Broussard P R, Tanaka C T, Nowak J, Moodera J S, Barry A and Coey J M D 1998 Science 28285

[14] Piano S, Bobba F, De Santis A, Giubileo F, Scarfato A and Cucolo A M 2006 Journal of Physics: Conference Series $\mathbf{4 3} 1123$

[15] Fominov Y V, Chtchelkatchev N M and Golubov A A 2002 Phys. Rev. B 66014507

[16] Tagirov L R1998 Physica C 307145

[17] Garifullin I A, Tikhonov D A, Garifyanov N N, Fattakhov M Z, Tagirov L R, Theis-Bröhl K, Westerholt K and Zabel H 2004 Phys. Rev. B 7054505 and references therein.

[18] Sidorenko A S, Zdravkov V I, Kehrle J, Morari R, Obermeier G, Gsell S, Schreck M, Müller C, Kupriyanov M Yu, Ryazanov V V, Horn S, Tagirov L R and Tidecks R 2009 JETP Lett. 90/2 139 [2009 Pis'ma v ZhETF 90 149] and references therein 
[19] Cirillo C, Rusanov A, Bell C and Aarts J 2007 Phys. Rev. B 75174510

[20] Cirillo C, Prischepa S L, Salvato M, Attanasio C, Hesselberth M and Aarts J 2005 Phys. Rev. B 72144511

[21] Kontos T, Aprili M, Lesueur J, Genet F, Stephanidis B and Boursier R 2002 Phys. Rev. Lett. 89 137007

[22] Kontos T, Aprili M, Lesueur J and Grison X 2001 Phys. Rev. Lett. 86, 304

[23] Crétinon L, Gupta A K, Sellier H, Lefloch F, Fauré M, Buzdin A and Courtois H 2005 Phys. Rev. B 72, 024511

[24] Cirillo C, Bell C, Iannone G, Prischepa S L, Aarts J and Attanasio C 2009 Phys. Rev. B 80 094510

[25] Cirillo C, Ilyina E A and Attanasio C 2011 Supercond. Sci. Technol. 24024017

[26] Kushnir V N, Prischepa S L, Aarts J, Bell C, Cirillo C and Attanasio C 2011 Eur. Phys. J. B 80 445

[27] Arham H Z, Khaire T S, Loloee R, Pratt W P Jr. and Birge N O 2009 Phys. Rev. B 80144515 and references therein

[28] Sharvin Yu V 1965 Sov. Phys. JEPT 21655 [1965 Zh. Eksp. Teor. Fiz. 48 984]

[29] Minhaj M S M, Meepagala S, Chen J T and Wenger L E 1994 Phys. Rev. B 4915235

[30] Rajanikanth A, Kasai S, Ohshima N and Hono K 2010 Appl. Phys. Lett. 97022505

[31] Heslinga D R, Shafranjuk S E, van Kempen H and Klapwijk T M 1994 Phys. Rev. B 4910484

[32] Xiong P, Xiao G and Laibowitz R B 1993 Phys. Rev. Lett. 711907

[33] Srikanth H and Raychaudhuri A K 1992 Phys. Rev. B 45383

[34] Volkov A F and Klapwijk T M 1992 Phys. Lett. A 168217

[35] Bakaul S R, Li K, Han G and Wu Y 2008 IEEE Trans. On Magn. 442737

[36] Chen T Y 2009 Physica C 469521

[37] Kashiwaya S, Tanaka Y, Koyanagi M, Takashima H and Kajimura K 1995 Phys. Rev. B 511350

[38] Krawiec M, Gyöffry B L and Annett J F 2004 Phys. Rev. B 70134519

[39] Wolf E L 1985 Principle of electron tunnelling spectroscopy, Oxford University Press

[40] Pannetier B and Courtois H 2000 Journ. of Low Temp. Phys. 118599 
[41] Nguyen C, Kroemer H and Hu E L 1992 Phys. Rev. Lett. 692847

[42] Strijkers G J, Ji Y, Yang F Y, Chien C L and Byers J M 2001 Phys. Rev. B 63104510 